\title{
Individual Differences in Amygdala and Ventromedial Prefrontal Cortex Activity are Associated with Evaluation Speed and Psychological Well-being
}

Article

Published Version

Van Reekum, C. M., Urry, H. L., Johnstone, T., Thurow, M. E., Frye, C. J., Jackson, C. A., Schaefer, H. S., Alexander, A. L. and Davidson, R. J. (2007) Individual Differences in Amygdala and Ventromedial Prefrontal Cortex Activity are Associated with Evaluation Speed and Psychological Well-being. Journal of Cognitive Neuroscience, 19 (2). pp. 237-248. ISSN 0898929X doi: https://doi.org/10.1162/jocn.2007.19.2.237 Available at https://centaur.reading.ac.uk/4382/

It is advisable to refer to the publisher's version if you intend to cite from the work. See Guidance on citing.

Published version at: http://dx.doi.org/10.1162/jocn.2007.19.2.237

To link to this article DOI: http://dx.doi.org/DOI:10.1162/jocn.2007.19.2.237

Publisher: M I T Press

Publisher statement: (02005 by The University of Chicago and The MIT Press. No permission is needed for noncommercial use or redistribution of any part of this publication as long as a complete citation is provided.

All outputs in CentAUR are protected by Intellectual Property Rights law, including copyright law. Copyright and IPR is retained by the creators or other copyright holders. Terms and conditions for use of this material are defined in 
the End User Agreement.

www.reading.ac.uk/centaur

\section{CentAUR}

Central Archive at the University of Reading

Reading's research outputs online 


\title{
Individual Differences in Amygdala and Ventromedial Prefrontal Cortex Activity are Associated with Evaluation Speed and Psychological Well-being
}

\author{
Carien M. van Reekum, Heather L. Urry*, Tom Johnstone, \\ Marchell E. Thurow, Corrina J. Frye, Cory A. Jackson**, \\ Hillary S. Schaefer, Andrew L. Alexander, \\ and Richard J. Davidson
}

\begin{abstract}
Using functional magnetic resonance imaging, we examined whether individual differences in amygdala activation in response to negative relative to neutral information are related to differences in the speed with which such information is evaluated, the extent to which such differences are associated with medial prefrontal cortex function, and their relationship with measures of trait anxiety and psychological well-being (PWB). Results indicated that faster judgments of negative relative to neutral information were associated with increased left and right amygdala activation. In the prefrontal cortex, faster judgment time was associated with relative decreased activation in a cluster in the ventral anterior cingulate cor-
\end{abstract}

\section{INTRODUCTION}

Vast differences exist in how individuals react to emotional information. Individual differences in emotion processing can be manifested either early in the processing stream, evidenced by an attentional bias to potentially significant information as displayed by highly anxious individuals (Mathews \& MacLeod, 2005; Mogg et al., 2000; Williams, Mathews, \& MacLeod, 1996), and/ or later in the processing stream, in what has been termed a "negativity bias" (Cacioppo \& Gardner, 1999; Taylor, 1991) or interpretative biases (Mathews \& MacLeod, 2005), which determine how information is appraised in terms of one's goals and concerns (Frijda, 1986). Variable recruitment of activation in amygdala and regions in the prefrontal cortex (PFC), which are part of the circuitry important to emotional and social behavior, likely mediates such individual differences in emotion reactivity (Davidson, 2000).

University of Wisconsin-Madison

*Heather Urry is now at the Department of Psychology, Tufts University.

**Cory Jackson is now at the Department of Psychology, University of Wyoming. tex (ACC, BA 24). Furthermore, people who were slower to evaluate negative versus neutral information reported higher PWB. Importantly, higher PWB was strongly associated with increased activation in the ventral ACC for negative relative to neutral information. Individual differences in trait anxiety did not predict variation in judgment time or in amygdala or ventral ACC activity. These findings suggest that people high in PWB effectively recruit the ventral ACC when confronted with potentially aversive stimuli, manifest reduced activity in subcortical regions such as the amygdala, and appraise such information as less salient as reflected in slower evaluative speed.
Animal and human studies suggest that the amygdala plays a central role in both the detection of potentially threatening information and in visceral responding to such information, which underlies energy mobilization for potential action (for a review, see Davis \& Whalen, 2001). Regions in the PFC, particularly the ventromedial regions including ventral parts of the anterior cingulate cortex (ACC), have been implicated in emotion processing through their involvement in regulating vegetative and somatic states (Bechara, Damasio, Damasio, \& Lee, 1999; Mayberg, 1997). For instance, research has demonstrated that the ventromedial PFC (vmPFC) is involved in extinguishing and relearning associations between hedonic valence and stimulus properties (Phelps, Delgado, Nearing, \& LeDoux, 2004; Rolls, 1999). The animal literature also documents the strong anatomical interconnection between the vmPFC and the amygdala (Ghashghaei \& Barbas, 2002; Amaral, Price, Pitkanen, \& Carmichael, 1992). Thus, the vmPFC might play a key role in biasing the evaluation of potentially negative information and in down-regulating the amygdalar response.

Given the ubiquitous involvement of the amygdala in fear and anxiety-related processes, it is not surprising that individual differences in amygdalar responding to 
negative information predict trait negative affect, particularly trait anxiety (Etkin et al., 2004; Mathews, Yiend, \& Lawrence, 2004; Schaefer et al., 2002). Whether individual differences in amygdalar responses to negative information predict positive affect remains understudied. Davidson (2004) has argued that high psychological well-being (PWB) is related to effective top-down regulation of the amygdala and fast recovery from negative stimuli. Supporting the position that a positive affective style modulates amygdala functioning (via the PFC) to potentially salient information, Kim, Somerville, Johnstone, Alexander, and Whalen (2003) demonstrated that a positive interpretation of surprise faces, which are affectively ambiguous, is associated with lower right amygdala activation and higher activation in the vmPFC. Furthermore, the surprise-neutral signal difference in the vmPFC was inversely associated with signal difference in the amygdala. Urry et al. (2006) established activation in a similar region in the vmPFC to be inversely associated with left amygdala activation when people were asked to voluntarily decrease negative affect. Furthermore, activation in the vmPFC when suppressing negative affect was positively associated with an adaptive pattern of diurnal cortisol rhythms, whereas activation in the left amygdala was negatively associated with these cortisol rhythms. In addition, Gusnard and colleagues (2003) demonstrated that higher levels of "persistence," a personality trait reflecting perseverance even when rewards are infrequent or when experiencing frustration or fatigue (Cloninger, Przybeck, Svrakic, \& Wetzel, 1994), are associated with higher activation in regions of the orbito-frontal cortex, including the vmPFC, when viewing affective images embedded within a large number of neutral images. Given the role of the vmPFC in integrating cognitive and emotional information (Bechara et al., 1999) and in modulating amygdalar activity (Milad \& Quirk, 2002), and the empirical findings summarized above, we propose that higher levels of adaptive emotional behavior, as reflected in greater PWB in daily life, will be associated with successful recruitment of the vmPFC when confronted with negative information.

Such individual differences in the recruitment of the vmPFC and the amygdala are likely to have a measurable impact on the way in which individuals evaluate negative information. Using behavioral measures such as reaction times, a number of studies have established that discrepant fear-relevant pictures (of snakes or spiders) are detected faster than discrepant fear-irrelevant ones when embedded in a visual search task (Öhman, Flykt, \& Esteves, 2001), and that negative pictures high in arousal are evaluated faster than negative pictures low in arousal (Robinson, Storbeck, Meier, \& Kirkeby, 2004). Importantly, research has also established strong individual differences in behavioral outcomes, where high trait-anxious compared to low trait-anxious people show facilitated processing of threatening information (Mogg
\& Bradley, 1999; Williams et al., 1996) and an interpretative bias toward negative connotations (Calvo \& Castillo, 2001). Furthermore, Robinson, Vargas, Tamir, and Solberg (2004) demonstrated that participants who were fast to evaluate negative words reported higher negative affect, lower life satisfaction, and more somatic symptoms in daily life compared with those who were slower to respond. The time taken to evaluate negative information thus provides a valid indicator of biases to interpret information negatively, in addition to actual evaluative outcomes. Given the role of the amygdala in evaluating the emotional significance of information and in mobilizing energy to prepare for potential action, variability in amygdalar activation should be predictive of faster response times to potentially negative information. Conversely, given the findings summarized earlier, a lowered bias toward negative information, reflected in longer judgment times, should then be related to increased vmPFC activation for negative relative to neutral information.

The aim of this study was to assess the extent to which individual differences in the response of the amygdala and the vmPFC to negative emotional information are related to the speed at which such information is deliberately evaluated, and their relationship with trait anxiety and PWB. We predicted that if higher amygdalar activation is related to a negativity bias in evaluating information, the negative valence of the information should be less equivocal and, therefore, the information should be evaluated faster as negative. Individuals who are biased toward interpreting information as negative, such as those high in trait anxiety, should thus exhibit stronger amygdalar responses to negative information and should be faster to evaluate the information as negative relative to individuals low in trait anxiety. Conversely, we predicted that individuals who report high PWB would be successful in down-regulating the amygdala (by way of the vmPFC), thereby rendering the information less negative to them, and thus, would be slower to evaluate the information as negative. To test these predictions, we used functional magnetic resonance imaging (fMRI) to measure brain activation in the amygdala and the PFC as participants evaluated complex negative and neutral pictures. We measured the time it took for participants to judge the valence of the pictures using a binary forced-choice affective decision task. We then assessed whether these measures of brain activation and behavioral outcome were predicted by trait anxiety and PWB.

\section{METHODS}

The data that are presented here are part of a larger study in which participants were asked to voluntarily regulate (either increase or decrease) affect in response to negative pictures. The instruction to regulate or to attend to the pictures was always presented $4 \mathrm{sec}$ into the picture presentation, which was after the person provided their 
valence judgment of the image. The regulation task is thus fully independent from the affective judgment task. In this report, we focus on the conditions where people were asked to simply attend to the negative and neutral images, and not regulate their responses to these images.

\section{Participants}

Twenty-nine participants (18 women, ages 61-65 years, $M=63.5$ years) were recruited by placing advertisements in a local newspaper. In addition to serving the scientific aims described in the Introduction, this effort also served as a feasibility study for recruiting participants from the Wisconsin Longitudinal Study, a larger longitudinal study of older adults in which the aim was to investigate the neural bases of voluntary emotion regulation (see Urry et al., 2006). As a result, we recruited participants of the same age. Due to technical problems, the data of 3 out of 29 participants could not be used. Furthermore, the data of one participant were excluded due to responding with only "neutral" to the picture judgment task. The data of the remaining 25 participants were further considered for data analysis. All participants were right-handed, and none had a history of or currently had a neurological illness. None of the participants suffered from claustrophobia or had any muscular or back problems that would prevent them from lying in the scanner for more than 1 hour. Participants received $\$ 70$ for their participation in the MRI session, and all participants gave informed consent prior to the administration of any of the procedures. All procedures were approved by the University of Wisconsin-Madison Health Sciences IRB.

\section{Stimuli}

We used a randomized event-related paradigm in which we presented one of two sets of digital color photographs $(800 \times 600$ resolution $)$ selected from the International Affective Picture System (IAPS; Lang, Bradley, \& Cuthbert, 1997) to each participant in the scanner. Seventy-two negative photos were selected according to the IAPS norms to be both unpleasant $(1=$ most unpleasant to $9=$ most pleasant; $M=2.35, S D=0.57)$ and arousing $(1=$ least arousing to $9=$ most arousing; $M=$ $5.82, S D=0.80)$, whereas 24 neutral photos were selected to be neither pleasant nor unpleasant $(M=5.04$, $S D=0.29)$, and nonarousing $(M=3.14, S D=0.79)$ (means and standard deviations represent aggregations across the two sets of images). The negative images depicted scenes of (potential) violence, attack, graveyards, sick animals, and close-ups of animals such as cockroaches, snakes, spiders, and sharks. Neutral images included pictures of neutral-looking faces of people, traffic scenes, household objects, mushrooms, and abstract art. The negative and neutral images had similar levels of perceptual complexity, as determined by computing the .jpeg image file size ${ }^{1}$ (negative: $M=206,354 \mathrm{kB}$, $S D=46,550$; neutral: $M=206,915 \mathrm{kB}, S D=43,418$; $t=-0.064 ; p=.948)$. Picture presentation order was pseudorandomized with the constraint that no more than five pictures of the same valence were shown consecutively. Stimulus presentation was accomplished using E-Prime software (Psychology Software Tools, Pittsburgh, PA), whereas visual stimulation was delivered via a fiber-optic goggle system (Avotec, Stuart, FL).

\section{Trial Structure}

A white fixation cross was depicted in the center of a black screen for $1 \mathrm{sec}$, coupled with a simultaneous tone to ensure attention to the upcoming trial. A picture was then presented for $8 \mathrm{sec}$. An auditory cue instructing the participant (e.g., "attend") was delivered through headphones $4 \mathrm{sec}$ after picture onset. Participants were instructed to continue following the task instruction, also after picture offset, until they were cued by an auditory instruction to "relax." This relaxation instruction was presented 4 sec after picture offset. The ensuing intertrial interval varied from 4 to $7 \mathrm{sec}$, permitting a proper estimation of the impulse response function. Trials ranged from 17 to $20 \mathrm{sec}$ duration.

\section{Task and Procedure}

Participants were instructed to indicate whether they evaluated a picture as negative or neutral by pressing one of two buttons on a button box as soon as the picture appeared, and before the "attend" instruction was provided. Responses provided during or after the instruction were not recorded.

A day prior to the scanning session, participants underwent a separate simulation session to acquaint them with the scanning environment and to train on performing the task inside the bore of an inactive MRI scanner shell. The real scan occurred on the morning following the simulation session. This scan session was divided into 4 blocks of 24 trials. Prior to the scan, the experimenter reminded the participant of the task instructions.

Once the scan session was over, participants were led into a separate room, where they provided subjective ratings of valence and arousal on half of the set (12 per condition) of the experimental picture stimuli seen in the scanner. Each picture was presented for $6 \mathrm{sec}$, after which the participant was cued to provide first their valence rating followed by the arousal rating.

\section{Data Acquisition and Reduction}

\section{Judgment Times}

Measures of judgment time were derived by calculating the difference between the onset time of the image and the participant's response. Judgment times shorter 
than 150 msec were excluded. To mirror the fMRI data reduction, judgment times of those trials for which pupil dilation (see below) was missing for over 50\% of the picture time, likely indicating trials during which participants had their eyes closed or otherwise did not optimally view the picture, were excluded as well, a total of 5.4\% of the trials. Proportional agreement scores were calculated by counting the number of observations for which the participant's response corresponded to the valence of the picture as derived from the normative ratings, divided by the total number of valid observations.

\section{Measures of Anxiety and Well-being}

Self-report questionnaires, including the 20 item StateTrait Anxiety Inventory (STAI-X; Spielberger, Gorsuch, Lushene, Vagg, \& Jacobs, 1983; $M=31.59$, $S D=6.86$, Cronbach's $\alpha=.87$ in our sample), the 11 item General Distress: Anxious Symptoms $(M=14.24, S D=3.24, \alpha=$ $.77)$, the 17 -item Anxious Arousal subscale $(M=20.69$, $S D=3.87, \alpha=.73$ ) of the Mood and Anxiety Symptom Questionnaire (MASQ; Watson \& Clark, 1991), and the 84-item Scales of Psychological Well-Being (Ryff, 1989; $M=72.19, S D=6.0, \alpha=.79$ ), were sent to the participants, who completed them prior to the laboratory visit. To maximize statistical power and to reduce the probability of Type I errors, we computed a composite measure of trait anxiety $(\alpha=.80)$, by calculating $z$-scores across subjects for the STAI-X and the two anxiety-related subscales of the MASQ, and then averaged the $z$-scored scales. A total PWB score was computed by taking the average of the six subscales of 14 items each, including Positive Relationships with Others $(M=71.25, S D=$ 10.96, $\alpha=.88)$, Autonomy $(M=69.33, S D=8.83, \alpha=$ .80), Environmental Mastery $(M=72.29, S D=8.07$, $\alpha=.77)$, Personal Growth $(M=76.38, S D=6.78, \alpha=$ $.84)$, Purpose in Life $(M=74.17, S D=6.83, \alpha=.76)$, and Self-Acceptance $(M=71.29, S D=8.67, \alpha=.82)$.

\section{Picture Ratings}

Participants rated the valence and arousal of half of the picture stimuli outside the scanner. For the valence ratings, participants were asked how pleasant or unpleasant they found each picture, using 9-point Likert scales, with 1 representing "very unpleasant" and 9 "very pleasant." For the arousal ratings, participants were asked to report how calm or excited/keyed up they felt in response to the picture on the 9-point scale where 1 represented "very calm" and 9 "very excited/keyed up." These valence and arousal ratings were averaged across the 12 observations per condition.

\section{Magnetic Resonance Imaging}

Images were acquired on a General Electric (Fairfield, CT) SIGNA 3.0-Tesla high-speed imaging device with a quadrature head coil. Functional images consisted of 30 interleaved 4-mm sagittal T2*-weighted echo-planar imaging (EPI) slices covering the entire brain (1 $\mathrm{mm}$ interslice gap; $64 \times 64$ in-plane resolution; $240 \mathrm{~mm}$ field of view [FOV]; 2000 msec repetition time; 30 msec echo time (TE); $60^{\circ}$ flip angle; 244 image volumes per run). Four EPI images with identical acquisition parameters but with TEs of 30, 31, 33, and $36 \mathrm{msec}$, respectively, were also acquired, and were used in calculating magnetic field maps for image distortion correction. In addition, we used an eyetracking device (SensoMotoric Instruments, Teltow, Germany) interfaced with an fMRIcompatible fiber-optic goggle system to monitor pupil dilation. In this study, measures of pupil dilation were used to identify trials for which the participant was likely not fully attending to the picture. Functional images were collected in four runs of approximately 8 minutes each. Immediately following acquisition of functional images, a three-dimensional T1-weighted inversion recovery fast gradient-echo scan was acquired $(256 \times 256$ in-plane resolution; $240 \mathrm{~mm}$ FOV; $124 \times 1.2 \mathrm{~mm}$ axial slices).

Analysis of fMRI data was performed with the Analysis of Functional NeuroImages software suite (AFNI v. 2.40e; Cox, 1996). After discarding the first five images collected for each of the four runs during reconstruction, the images were time-corrected for slice acquisition order, and motion-corrected registering all the time points to the last time point of the last run. Each run was then high-pass filtered at $0.02 \mathrm{~Hz}$ to remove slow drift. In-house software was used to correct for image distortion at each time point based on the calculated field maps.

We then computed single-subject GLMs to estimate the hemodynamic response for each of the conditions using a deconvolution procedure that specified a predictor for each second, for a total of $20 \mathrm{sec}$ starting at picture onset. Six predictors (three translation, three rotation) based upon estimated motion were also included to model possible variance due to motion. Trials for which the pupil dilation data were missing for more than 50\% of the total trial length, indicating that the participant was not fully attending to the picture, were eliminated, as were time points where estimated motion peaks exceeded $1.5 \mathrm{~mm}$. These criteria resulted in $5.4 \%$ of trials being deleted. Percent signal change was calculated for each time point (100* beta coefficient/baseline), and then an area-under-the-curve (AUC) metric was calculated by summing the percent signal change values across an 8 -sec window (7th-14th time points inclusive after picture onset). This 8-sec window maximizes chances of capturing the peak of the hemodynamic response even if the peak varies temporally as a function of brain region and/or condition. These estimates of AUC for percent signal change were transformed into Talairach space and spatially blurred with a 5-mm full-width half-maximum Gaussian filter. 
Mean AUC percent signal change estimates across all voxels in two (left and right) amygdala regions of interest (ROIs), defined using the Talairach Atlas provided with AFNI, were extracted for each subject for further analysis in SPSS 12 (SPSS, Chicago IL). To examine the role of prefrontal regions in judging negative images, we conducted an across-subjects voxelwise regression analysis entering judgment times (negative neutral) as a predictor of brain activation (negative neutral). We limited the search space to the medial, superior, middle, and inferior frontal gyri, the anterior cingulate, orbital, and rectal gyri, as derived from the Talairach Atlas provided with AFNI (total 42,164 voxels). Clusters of activation within this search space were identified using Monte Carlo simulations (AFNI's AlphaSim procedure) to achieve a corrected cluster threshold of $p<.05$, which corresponded to a cluster size of at least $304 \mathrm{~mm}^{3}$. Mean AUC percent signal change estimates, computed across all voxels in each of these clusters, were extracted for each subject for further analysis in SPSS 12.

\section{RESULTS}

\section{Judgment Times}

The average latencies to make evaluative decisions on the valence of the picture were computed for each participant, excluding judgment times shorter than $150 \mathrm{msec}(0.3 \%$ of the total number of valid observations). The difference between the judgment times to the negative pictures $(M=1905.2, S D=389.4)$ and to the neutral pictures $(M=1697.9, S D=322.0)$ was significant, $t(24)=2.68, p=.013$. The proportional agreement score, that is, the proportion of trials on which the individual's evaluative decision corresponded with our categorization of the pictures based upon the normative ratings, was lower for the negative $(M=$ $0.78, S D=0.20)$ than for the neutral pictures $(M=$ $0.96, S D=0.09)$, suggesting that overall the negative images were less clear-cut negative than the neutral images were neutral, for at least a majority of participants. The correlation between the agreement scores and judgment times suggested that the higher the proportional agreement score, the less time it took for participants to judge the negative pictures as negative, $r=-.48, n=25, p=.016$. The correlation between the agreement score and the time it took to judge neutral pictures, on the other hand, was not significant, $r=-.04, p>.80$ (but note that the agreement score of neutral is close to ceiling and has low variability). Thus, people who were faster to evaluate the negative pictures more often decided that the negative pictures were negative. Conversely, those people who evaluated more of the negative images as neutral took more time to evaluate the negative images.

\section{Amygdala}

In line with prior research, the Valence $\times$ Hemisphere multivariate analysis of variance performed on the AUC percent signal change revealed a main effect for valence $[F(1,24)=6.22, p=.02]$, with no interaction effect between valence and hemisphere, $F<1$. This effect confirmed that activation in both amygdalae was significantly greater in response to negative pictures $(M=1.17$, $S D=1.23$, and $M=1.16, S D=1.55$ for left and right amygdala, respectively) relative to neutral pictures $(M=$ $0.54, S D=1.56$, and $M=0.50, S D=1.54$ for left and right, respectively) (see Figure 1A for a depiction of the averaged estimated hemodynamic responses to negative and neutral pictures).

\section{Correlations between Judgment Times and the Amygdala}

We predicted that higher amygdala activation would be associated with faster evaluation speeds for negative relative to neutral images. To standardize the judgment times, we computed a negative - neutral difference score for each subject, and then correlated this score with left and right amygdala negative - neutral difference scores. As illustrated in Figure 1B, individuals who responded with stronger amygdalar activity to the negative (relative to the neutral) pictures were faster in responding to the negative (relative to the neutral) images, $r=-.46, n=25, p=.021$ for the left amygdala, and $r=-.44, n=25, p=.029$ for the right amygdala.

\section{Voxelwise Regression Analysis in the PFC with Judgment Time}

We also predicted that slower judgment times would be associated with higher vmPFC activation. To evaluate this hypothesis, we used the judgment time difference score (negative - neutral) as a predictor in a voxelwise regression analysis, limiting the search space to the PFC (see Methods section). We found two clusters that survived correction for multiple comparisons, one in the ventral ACC (BA 24; see Figure 2), and the other in the medial frontal gyrus (MFG, BA 6). After extracting mean percent signal AUC for each subject across all voxels of each cluster, we determined that the ventral ACC exhibited an opposite pattern to that of the amygdala, with negative - neutral judgment time being positively correlated with negative relative to neutral activity in this region, $r=.63, n=25, p=$ .001. The opposite pattern might suggest that participants showing higher signal difference in the ventral ACC would show lower signal difference in amygdala. However, activation in neither the left nor right amygdala was correlated with the ventral ACC, $r<.1, n=$ 
Figure 1. (A) Averaged estimated hemodynamic responses to negative (red) and neutral (blue) images extracted for the left and right amygdala a priori ROI, defined using the Talairach Atlas provided with AFNI. This a priori region has a total volume of $1288 \mathrm{~mm}^{3}$, and extends from $x=17$ to 29 (or -17 to -29 for the left amygdala ROI), $y=-11$ to 1 , and $z=-22$ to -8 . The responses depicted indicate higher activity in the left and right amygdala for negative compared to neutral information. The calculated AUC metric (which is a sum of the \% signal change measured at each timepoint) used in subsequent analyses is depicted in gray. (B) The scatterplots depict the correlation between judgment time (negative neutral) and mean difference in \% signal AUC across voxels in left and right amygdala regions, defined using the Talairach Atlas. Left and right amygdala activity is negatively associated with judgment time. neg $=$ negative; neu $=$ neutral .
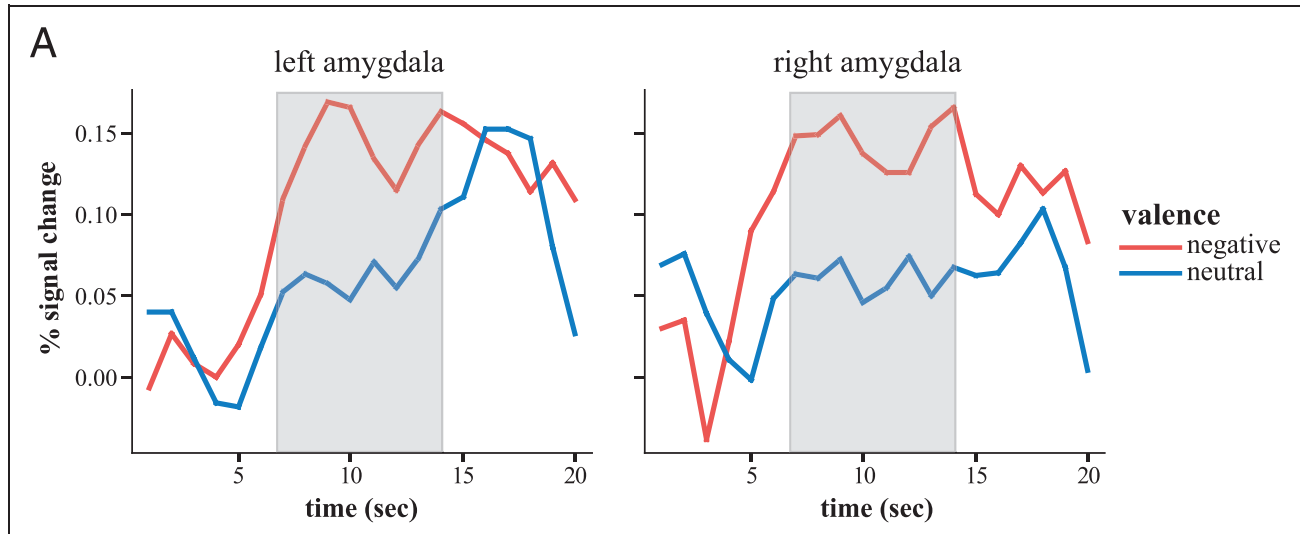

B
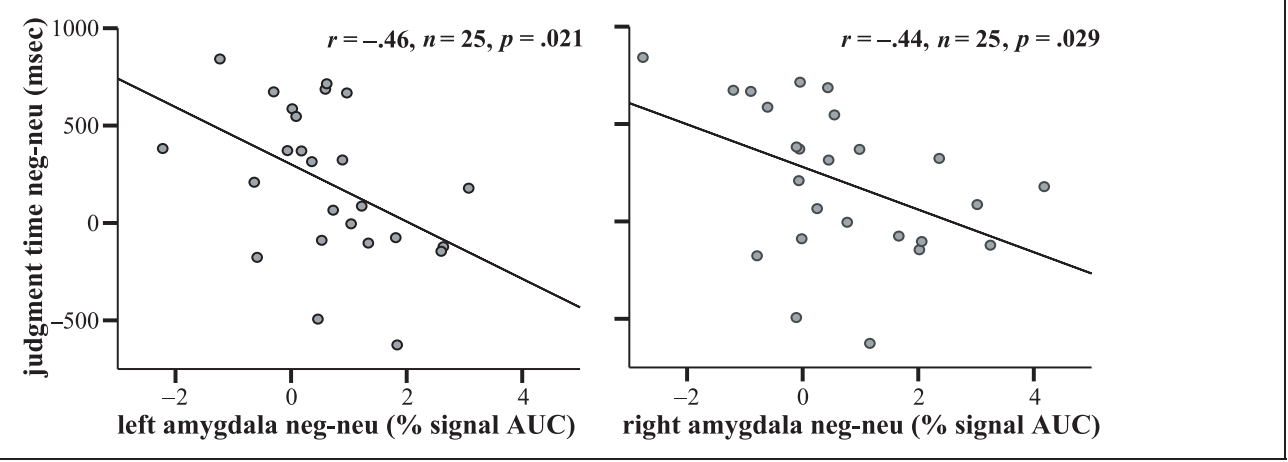

24 (after removal of one extreme value in the ventral ACC cluster). The correlation between the negative neutral signal difference in the MFG and judgment time was not significant, $r=.27, n=22, p=.22$ (original $r=.64, n=25, p=.001$; close examination of the scatterplot indicated that the correlation was carried by 3 of the 25 cases, which rendered the correlation spurious). We therefore excluded this frontal cluster from further analysis.

\section{Correlations with Anxiety and Well-being}

We next examined associations between judgment time and patterns of brain activation in the regions noted
Figure 2. Ventral ACC activity predicts judgment time. (A) Sagittal view of a cluster in the ventral ACC (BA 24, cluster volume of $368 \mathrm{~mm}^{3}$, max. $t=4.35$ at $x=-3, y=29$, and $z=-2$ in Talairach space) resulting from a voxelwise regression analysis. (B) The scatterplot depicts the positive correlation between judgment time and mean difference in $\%$ signal AUC across voxels in this cluster in the ventral ACC. The correlation remains significant when the case with an extremely high \% signal change value in the ventral ACC is removed, $r=.60$, $n=24, p=.002$. neg $=$ negative; neu $=$ neutral.

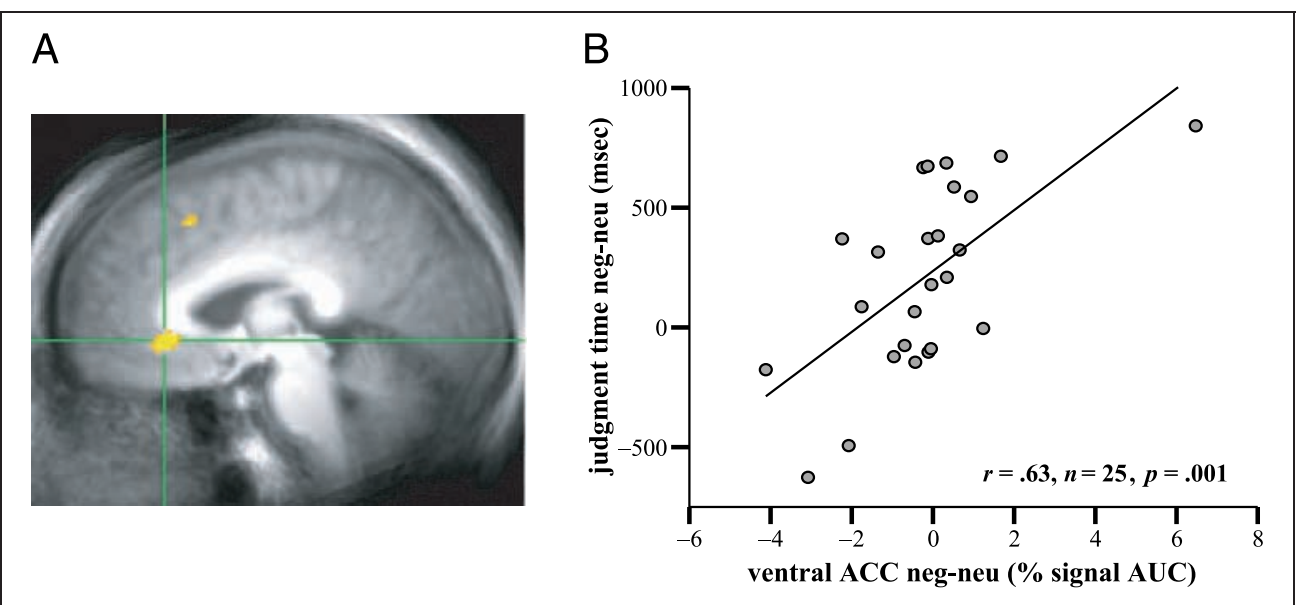


above, on the one hand, and our trait anxiety and $\mathrm{PWB}$ measures on the other. Suggesting modest overlap between these two measures, high reported trait anxiety was associated with lower PWB, $r=-.45, n=24, p=.028$.

\section{Trait Anxiety}

We predicted that higher trait anxiety would be associated with being faster to judge negative relative to neutral pictures and higher amygdala activation in response to negative relative to neutral pictures. Contrary to predictions, our composite measure of trait anxiety (see Methods section) was not significantly correlated with judgment time, $r=-.30, n=25, p=.146$. Furthermore, there were no significant correlations between judgment time and any of the three anxiety subscales (max. $r=-.32, p=.12$, between MASQ: anxious arousal and judgment times). Finally, the composite measure of trait anxiety did not significantly correlate with left or right amygdala signal (negative - neutral), nor with any of the subscales (all $r$ s $<.10$ ). Ventral ACC signal (negative - neutral) was not significantly correlated with the trait anxiety composite, nor with any of the anxiety subscales, $r=-.20, n=25, p=.33$ for trait anxiety, all $r \mathrm{~s}<.20$ for the anxiety subscales.

\section{Psychological Well-being}

We hypothesized that higher PWB would be associated with being slower to judge negative relative to neutral pictures, and with higher vmPFC activation in response to negative relative to neutral images. As predicted, total PWB was significantly associated with judgment time, $r=.43, n=24, p=.038$, such that people reporting high PWB were slower to judge the negative relative to neutral images. There was a particularly strong positive correlation between judgment time and the PWB Purpose in Life scale, $r=.63, n=23, p=.001$. Although amygdalar activity was not associated significantly with the total PWB score, the scores for the Purpose in Life subscale were inversely associated with the left amygdala, $r=-.45, n=23, p=.033$. Activation in the ventral ACC was strongly associated with total PWB, $r=.67$, $n=24, p<.001$, indicating that the higher the total PWB, the more activation in the ventral ACC for the negative, relative to the neutral, condition (Figure 3). Signal change in the ventral ACC was also significantly positively correlated with the Purpose in Life subscale, $r=.56, n=23, p=.005$.

\section{Regression Analyses}

We next performed a hierarchical regression analysis to assess whether variance in judgment time was explained by anxiety and well-being, by brain activation, or by both. To eliminate multicollinearity in our regres-

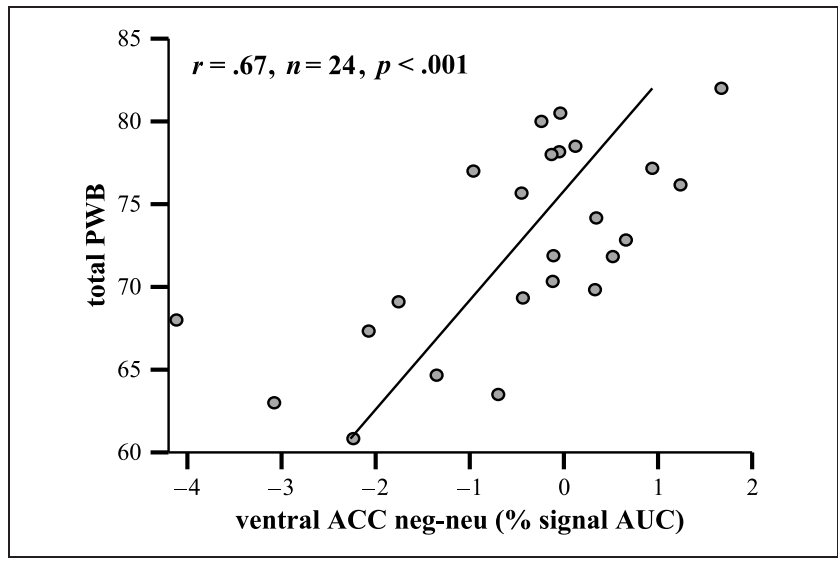

Figure 3. Activity in the ventral ACC in response to negative versus neutral images is positively associated with total PWB. Note that $n=24$ due to a missing observation in total PWB. neg $=$ negative; neu $=$ neutral.

sion model, we orthogonalized PWB and trait anxiety by residualizing each of these measures, removing shared variance. Furthermore, given the lack of a hemispheric effect and the high correlation between the left and the right amygdala $(r=.78)$, we averaged the left and right amygdala, thus obtaining a single measure of amygdalar activation. We entered residualized PWB and trait anxiety in a first step, and negative - neutral signal difference in the amygdala and ventral ACC in a second step.

In line with the correlational analyses, PWB significantly explained unique variance in judgment time $[\beta=.48$, $t(21)=2.17, p=.042]$, whereas trait anxiety did not $[\beta=$ $-.33, t(21)=-1.48, p=.153]$. However, the effects of these predictors were abolished when the measures of brain activation were entered in a second step $\left[\Delta R^{2}=\right.$ .33, $\Delta F(2,19)=6.42, p=.007]$. Both activation in the amygdala and ventral ACC explained unique variance in judgment time [amygdala: $\beta=-.37, t(19)=-2.3$, $p=.033$; ventral ACC: $\beta=.6, t(19)=2.79, p=.012]$, whereas PWB $(\beta<.10)$ and trait anxiety $[\beta=-.18$, $t(19)=0.96, p=.348]$ no longer did. To assess whether the ventral ACC significantly accounted for the relation between PWB and judgment time, we performed a test of collapsibility (Clogg, Petkova, \& Shihadeh, 1992; see MacKinnon, Lockwood, Hoffman, West, \& Sheets, 2002 for a comparison of the different tests of mediation). Results of this test are consistent with the idea that the ventral ACC indeed mediates the relation between PWB and judgment time, $t(21)=2.39, p=.026$.

\section{Picture Ratings}

The ratings of valence and arousal of the negative images provided by our participants after the scanning session for a subset of the pictures presented in the scanner corresponded with the normative ratings of the pictures used: $M=2.54, S D=1.4$ for the valence ratings compared to $M=2.35$ for the normative valence 
ratings; $M=5.37, S D=1.96$ for the arousal ratings versus $M=5.82$ for the normative ratings, all $n=25$. None of the correlations between valence and arousal ratings and judgment time or brain activation were significant, highest $r=.26, p=.21$.

\section{Correlations with Voluntary Regulation Conditions}

The results thus far have been calculated on the basis of trials in which subjects were instructed to "attend" to the picture without changing their response. However, in additional trials, participants were also instructed to regulate (enhance/increase or suppress/decrease) their response to a number of the negative pictures through reappraisal. Although the instruction to regulate or to attend to the picture always followed the judgment task, which was performed during the first 4 sec following picture onset, it remains nonetheless possible that the context of the regulation task affected the associations reported here. To determine whether the findings we have reported thus far are specific to the attend trials, we computed the correlations between the judgment times to all negative images (controlling for the judgment times to the neutral images) and the blood oxygen level dependent (BOLD) response in the left and right amygdala and the ventral ACC, collapsing across all regulation conditions. These correlations are almost identical to those reported for the attend condition (judgment time with the left amygdala: $r=-.47$, $p=.017$; with the right amygdala: $r=-.40, p=.045$; with the ventral ACC: $r=.62, p=.001$ ). The associations with PWB are similar to those reported for the attend condition as well, although the correlation between PWB Purpose in Life subscale and the amygdala is no longer significant (left amygdala: $r=-.34, p=.11$; right amygdala: $r=-.30, p=.17$ ). Similarly, the correlations computed for each of the enhance-neutral and suppress-neutral contrasts separately are identical in direction to those reported for the attend-neutral contrast, although in some cases no longer significant at the $p<.05$ threshold (but all $p<.1$, except for Purpose in Life and amygdala, where the lowest correlation drops to $r=-.18, p=.41$ ). Thus, the regulatory context does not affect the basic associations reported here.

Finally, to test whether those people with a high PWB also were more successful at voluntarily down-regulating the amygdala, we correlated the suppress-attend contrast in the left and right amygdalar response with PWB. There was no significant association between total PWB and the left amygdala difference score $(r=.05, p=.81)$ or the right amygdala $(r=.19, p=.39)$, nor between Purpose in Life and the left amygdala $(r=.25, p=.26)$, or the right amygdala $(r=.09, p=.68)$. Parallel analyses between the suppress-attend difference score in the ventral ACC and the left and right amygdala were in the expected direction, but not significant (the ventral
ACC with the left amygdala: $r=-.14, p=.50$ and the ventral ACC with the right amygdala: $r=-.18, p=.38$ ). Thus, there is no indication that the associations between PWB and amygdalar and ventral ACC response to negative images are related to individual differences in voluntarily regulating negative affect. The associations thus seem to exist at the level of the initial appraisal of potentially negative stimuli.

\section{DISCUSSION}

Individual differences in response to adversity represent a key attribute that in part governs PWB. The data we report indicate that individual differences in amygdala and ventral ACC activation in response to negative emotional stimuli predict both evaluation speed and PWB. Our results demonstrate that people who responded with stronger amygdalar BOLD signal for negative relative to neutral pictures evaluated negative relative to neutral pictures faster. On the other hand, people who responded with stronger ventral ACC BOLD signal for negative relative to neutral pictures evaluated the negative relative to neutral pictures slower. Furthermore, PWB, but not trait anxiety, predicted unique variance in judgment time, where higher levels of PWB were associated with longer judgment time. PWB was strongly associated with activation measured in the ventral ACC where higher PWB was associated with more activation in the ventral ACC for negative relative to neutral information. Moreover, signal differences observed in the ventral ACC significantly accounted for the relationship between judgment time and PWB. Finally, individuals who responded with higher amygdalar activation reported lower purpose in life, a facet of PWB. The Purpose in Life subscale was positively associated with judgment time and activation in the ventral ACC. Trait anxiety was not associated with amygdala or ventral ACC activation.

In our study, we conceptualized the time people take to evaluate negative compared to neutral information as an indicator of an individual's relative interpretative bias. On average, the judgment times to the negative pictures were longer than to the neutral pictures. However, it is important to note that we observed large individual differences in judgment time, with some individuals being as much as half a second slower or faster to evaluate the negative pictures relative to the neutral ones. The proportion agreement score was, on average, lower for the negative than for the neutral pictures. This suggests that, on average, participants were less certain of their affective decision for the negative images. We correlated the proportion agreement score with the judgment times and found that people who evaluated the negative relative to neutral pictures quickly more often rated the negative picture as negative. Thus, uncertainty on how to affectively categorize an image results in longer evaluation times, as reflected in our data. 
Following Irwin et al. (1996), a large number of fMRI studies have demonstrated higher amygdala activity in response to negative versus neutral complex pictures similar to the ones we used (for a meta-analysis, see Baas, Aleman, \& Kahn, 2004). We replicated these findings. Importantly, our study demonstrates that individual differences in amygdala reactivity to negative information, taking into account the response to neutral information, is predictive of the time people took to evaluate the information. The association between amygdala activity and judgment times supports our conceptualization of judgment time being an indicator of an individual's bias in interpretation. Given recent demonstrations of associations between amygdala activity and increased arousal, both subjectively rated (Phan et al., 2004) as well as autonomic arousal indexed by measures of electrodermal activity or heart rate, in response to emotionally salient information (Dalton, Kalin, Grist, \& Davidson, 2005; Critchley, Mathias, \& Dolan, 2002; Phelps et al., 2001; Williams et al., 2001), our behavioral effects may be mediated by a stronger affective response to the negative stimuli for those individuals with stronger amygdalar activation.

Interestingly, individual differences in the actual evaluative outcome (i.e., the binary evaluative decision while in the scanner as well as the valence and arousal ratings measured after the scan session) were not correlated with variation in amygdalar activation. The binary assessment of image valence (negative or neutral), and the postscan ratings provided after a 6-sec presentation of the image, likely capture the outcomes of a number of processes including social desirability and demand characteristics in addition to the appraisal of the information. Judgment time, on the other hand, captures the duration rather than the outcome of an evaluative process. As Whalen (1998) has suggested, the amygdala is probably less implicated in the outcome of affect processing such as felt affect, but rather modulates affect processing by biasing neural encoding in cortical networks. Following this line of reasoning, our data suggest that judgment time is a more sensitive index of an evaluative process than measures of affect outcome, such as ratings of valence and arousal, hence, covaries with amygdala activity.

Our findings further indicate that activation in the ventral ACC is positively associated with the time it takes to judge negative relative to neutral information. This pattern of association with judgment time is thus opposite to that of the amygdala. The ventral ACC and the amygdala form part of an anatomical circuit that has been implicated in emotion and emotion regulation. In an independent study with an entirely separate group of subjects, Urry et al. (2006) recently demonstrated inverse coupling between vmPFC and amygdala activity when participants were instructed to decrease experienced negative affect through reappraisal of negative information. Kim et al. (2003) found an inverse association between activation in the amygdala and the vmPFC in response to surprise faces, with individuals who re- sponded with higher BOLD responses in the vmPFC and lower responses in the amygdala rating the surprise faces more positively. Our data thus suggest that people who are effective at recruiting this area of the ventral ACC and modulating the amygdala exhibit a lowered negativity bias. However, in this study, we found no direct association between BOLD signal in the amygdala and the ventral ACC, either when evaluating the information or when participants were instructed to decrease experienced negative affect through reappraisal. The reason for this lack of association is unclear, but it appears that whether or not an inverse coupling is observed between these regions is highly task-dependent. For example, in their follow-up study to Kim et al. (2003), Kim and colleagues (2004) similarly did not observe an inverse association between the amygdala and the vmPFC in response to surprise faces that were presented within an explicit positive or negative context. They suggest that the presence of contextual affective cues may have reduced the ambiguity of their surprised faces or may have rendered the affective valence more explicit, thus alleviating the need for vmPFC mediation. In the current study, the explicit evaluation of potentially emotioneliciting information as negative or neutral, and evaluating this information prior to the voluntary regulation instruction, may similarly have rendered the information less ambiguous, and therefore, reduced any inverse coupling between the amygdala and the ventral ACC. This notion requires explicit testing in future research.

Having observed a pattern of significant associations between judgment time on the one hand, and amygdala and ventral ACC activity on the other hand, one can ask whether a bias in interpreting negative information is associated with higher trait anxiety and/or lower PWB. Our data suggest that PWB predicts unique variance in judgment time, whereas trait anxiety does not. Furthermore, we predicted associations between anxiety and well-being on the one hand and amygdala and vmPFC activity on the other hand. Interestingly, individual differences in trait anxiety were not significantly associated with either amygdalar or ventral ACC activation in response to negative versus neutral images, but individual differences in PWB were strongly associated with ventral ACC activity. We further demonstrated that variation in ventral ACC activity significantly accounted for the association between PWB and judgment time, suggesting that individuals high in PWB activated the ventral ACC more in response to negative relative to neutral information, and individuals who activate the ventral ACC more displayed a lower negative evaluative bias when evaluating negative relative to neutral information.

The literature on well-being suggests that there are two distinct types of well-being, eudaimonic and hedonic well-being. As stated by Ryff (1989), eudaimonic wellbeing is characterized by the realization of one's potential, whereas hedonic well-being is more focused on the experience of happiness and satisfaction. A recent study 
involving aging women suggests that these more existential, active aspects of well-being are associated with measures of endocrine, immunological, and cardiovascular functioning, whereas hedonic well-being was not (Ryff, Singer, \& Love, 2004). Furthermore, a recent study suggests that PWB is, to a large extent, distinct from, and not mirroring, a negative affective style including trait anxiety in its associations with health-related measures of the neuroendocrine system and of cardiovascular risk (Ryff et al., 2006). Our study suggests as well that PWB and trait anxiety, although correlated, are not necessarily mirrored in their associations with brain function when evaluating negative information.

Although amygdala activation was not associated with total PWB, activity in the left amygdala was associated with purpose in life. This subscale captures the extent to which people report having a goal and a sense of direction in life (Ryff, 1989). The purposeful, more active nature of well-being captured by this scale may well be the reason that this subscale, in particular, is correlated with left amygdalar activation, given the role of the amygdala in activating the peripheral nervous system to ready the organism for action. Having said that, high interconnectivity with more dorsal/lateral areas of the PFC, areas that underlie cognitive control and goaldirected behavior (Miller \& Cohen, 2001), and with systems crucial for somatic output (Mayberg, 1997) may explain why, ultimately, the ventral ACC, more than the amygdala, contributes to high levels of PWB.

The relative lack of significant associations between our behavioral and brain findings on the one hand and trait anxiety on the other is puzzling. It is worth noting that past research documenting associations between anxiety and changes in the amygdala and/or areas of the PFC often measured activation in these areas in response to a threat of shock, fearful facial expressions, or words specific to the object of people's anxiety (Bishop, Duncan, Brett, \& Lawrence, 2004; Bishop, Duncan, \& Lawrence, 2004; Etkin et al., 2004; Simpson, Drevets, Snyder, Gusnard, \& Raichle, 2001). This includes a recent study from our laboratory where high trait-anxious people activated regions in the vmPFC less, and showed more intense negative affect indexed with corrugator activity in a threat-of-shock paradigm (Shackman et al., 2005). It is possible that trait anxiety and activation in amygdala and vmPFC might be coupled specifically when individuals are presented with anxietyrelated stimuli, and less with images that may evoke a number of affective states involving, among others, anger, disgust, or sadness in addition to fear/threat. Hence, studies examining individual differences in brain systems underlying processing of negative affect, in particular when the study does not incorporate fear-related stimuli per se, should incorporate measures of positive affect, such as PWB, to capture variance possibly left unexplained by variation in negative affective style such as trait anxiety.
Although this study was not focused on the effects of age on emotion processing per se, the participants were older (average age of 63.5 years) than the more commonly recruited college-aged participants. Both structural and functional differences have been found to underlie age effects in cognitive decrements, with the strength of association between age and brain structure/ function being variable across brain regions and task type: Across two studies, Raz et al. (1997, 2004) demonstrate the lateral PFC to be most vulnerable to the effects of aging, with decline also being observed for the hippocampus and areas in the parietal cortex, whereas the ACC was not significantly correlated with age in either study. These findings were replicated by Grieve, Clark, Williams, Peduto, and Gordon (2005) using voxelbased morphometry, and evidence was also provided of comparative preservation of limbic and paralimbic areas including the amygdala.

With regard to brain function and aging, differences have been demonstrated in neural substrates underlying processing of emotional facial expressions, with older adults activating regions in the PFC, including Broca's area, more than younger adults, and younger adults activating limbic regions, including the amygdala, more than older adults, when performing an emotion discrimination task of emotional expressions (Tessitore et al., 2005; Gunning-Dixon et al., 2003). Recent research suggests that older adults (aged 70-90 years) rate negative images similar to those used here as less arousing, and respond with lesser amygdala activation to these negative images, compared to younger adults (aged 1829 years) (Mather et al., 2004). However, Mather and colleagues further demonstrate that when younger participants are selected on the basis of the similarity in arousal ratings to the older participants, the patterns of amygdalar activation become comparable, which suggests that arousal and amygdala activity in response to negative images are correlated, regardless of age. Note also that the participants in our study rated the negative images as negative and arousing as the normative group upon which the selection of the images was based. Furthermore, MacPherson, Phillips, and Della (2002) reported that older individuals show decrements in performance on neuropsychological tasks dependent on dorsolateral PFC function, but not on tasks involving emotion and social decision making that are dependent on vmPFC function. These findings imply that, although main effects in amygdalar or vmPFC responding or judgment times to evaluating negative information may differ across age groups, individual differences in activation in these brain areas and their association with judgment time and PWB likely generalize to all ages (see also Urry et al., 2006, for a discussion of effects of aging on brain function underlying voluntary emotion regulation). Future work should assess whether these associations are specific to an older age group by the inclusion of a younger comparison group. 
In conclusion, we demonstrated that individuals who are slower to evaluate negative relative to neutral information are characterized by higher levels of PWB and display higher levels of activity in the ventral ACC and lower levels of activity in the amygdala when confronted with negative relative to neutral information. These data lead us to speculate that this region of the vmPFC, which has been implicated in the down-regulation of amygdalar functioning in some contexts, plays a key role in goal-directed positive affect and well-being. People characterized by a positive affective style are likely to effectively recruit this brain region when confronted with potentially aversive information, show reduced activity in subcortical regions such as the amygdala, and (re)appraise such information as less salient while dampening bodily responses accompanying a readiness to act.

\section{Acknowledgments}

We thank Michael Anderle and Ron Fisher for their assistance with data collection and Jonghoon Lee for his assistance with image distortion correction. This work was supported by grants from the National Institutes of Mental Health (P50-MH52354, P50-MH069315, R37-MH43454), the National Institute on Aging (P01-AG021079), and by grant M01-RR03186 from the General Clinical Research Centers Program of the National Center for Research Resources, National Institutes of Health.

Reprint requests should be sent to Richard J. Davidson or Carien M. van Reekum, Laboratory for Affective Neuroscience, Dept. of Psychology, University of Wisconsin-Madison, 1202 W. Johnson St., Madison, WI 52706, or via e-mail: rjdavids@ wisc.edu, cmvanreekum@wisc.edu.

\section{Note}

1. A large file size indicates that the image is varied in color and contrast relative to a smaller file size (see Calvo \& Lang, 2004; Junghöfer, Bradley, Elbert, \& Lang, 2001 for a similar analysis).

\section{REFERENCES}

Amaral, D. G., Price, J. L., Pitkanen, A., \& Carmichael, S. T. (1992). Anatomical organization of the primate amygdaloid complex. In J. P. Aggleton (Ed.), The amygdala: Neurobiological aspects of emotion, memory and mental dysfunction (pp. 1-66). New York: Wiley-Liss.

Baas, D., Aleman, A., \& Kahn, R. S. (2004). Lateralization of amygdala activation: A systematic review of functional neuroimaging studies. Brain Research Reviews, 45, 96-103.

Bechara, A., Damasio, H., Damasio, A. R., \& Lee, G. P. (1999). Different contributions of the human amygdala and ventromedial prefrontal cortex to decision-making. Journal of Neuroscience, 19, 5473-5481.

Bishop, S., Duncan, J., Brett, M., \& Lawrence, A. D. (2004). Prefrontal cortical function and anxiety: Controlling attention to threat-related stimuli. Nature Neuroscience, 7, 184-188.

Bishop, S. J., Duncan, J., \& Lawrence, A. D. (2004). State anxiety modulation of the amygdala response to unattended threat-related stimuli. Journal of Neuroscience, 24, 10364-10368.
Cacioppo, J. T., \& Gardner, W. L. (1999). Emotion. Annual Review of Psychology, 50, 191-214.

Calvo, M. G., \& Castillo, M. D. (2001). Selective interpretation in anxiety: Uncertainty for threatening events. Cognition and Emotion, 15, 299-320.

Calvo, M. G., \& Lang, P. J. (2004). Gaze patterns when looking at emotional pictures: Motivationally biased attention. Motivation and Emotion, 28.

Clogg, C. C., Petkova, E., \& Shihadeh, E. S. (1992). Statistical-methods for analyzing collapsibility in regression-models. Journal of Educational Statistics, 17, 51-74.

Cloninger, C. R., Przybeck, T. R., Svrakic, D. M., \& Wetzel, R. D. (1994). Temperament and Character Inventory (TCI): A guide to its development and use. St. Louis: Center for Psychobiology of Personality, Washington University.

Cox, R. W. (1996). AFNI: Software for analysis and visualization of functional magnetic resonance neuroimages. Computers and Biomedical Research, 29, 162-173.

Critchley, H. D., Mathias, C. J., \& Dolan, R. J. (2002). Fear conditioning in humans: The influence of awareness and autonomic arousal on functional neuroanatomy. Neuron, 33, 653-663.

Dalton, K. M., Kalin, N. H., Grist, T. M., \& Davidson, R. J. (2005). Neural-cardiac coupling in threat-evoked anxiety. Journal of Cognitive Neuroscience, 17, 969-980.

Davidson, R. J. (2000). Affective style, psychopathology, and resilience: Brain mechanisms and plasticity. American Psychologist, 55, 1196-1214.

Davidson, R. J. (2004). Well-being and affective style: Neural substrates and biobehavioural correlates. Philosophical Transactions of the Royal Society of London, Series B, Biological Sciences, 359, 1395-1411.

Davis, M., \& Whalen, P. J. (2001). The amygdala: Vigilance and emotion. Molecular Psychiatry, 6, 13-34.

Etkin, A., Klemenhagen, K. C., Dudman, J. T., Rogan, M. T., Hen, R., Kandel, E. R., et al. (2004). Individual differences in trait anxiety predict the response of the basolateral amygdala to unconsciously processed fearful faces. Neuron, 44, 1043-1055.

Frijda, N. H. (1986). The emotions. Cambridge: Cambridge University Press.

Ghashghaei, H. T., \& Barbas, H. (2002). Pathways for emotion: Interactions of prefrontal and anterior temporal pathways in the amygdala of the rhesus monkey. Neuroscience, $115,1261-1279$.

Grieve, S. M., Clark, C. R., Williams, L. M., Peduto, A. J., \& Gordon, E. (2005). Preservation of limbic and paralimbic structures in aging. Human Brain Mapping, 25, 391-401.

Gunning-Dixon, F. M., Gur, R. C., Perkins, A. C., Schroeder, L. Turner, T., Turetsky, B. I., et al. (2003). Age-related differences in brain activation during emotional face processing. Neurobiology of Aging, 24, 285-295.

Gusnard, D. A., Ollinger, J. M., Shulman, G. L., Cloninger, C. R., Price, J. L., Van Essen, D. C., et al. (2003). Persistence and brain circuitry. Proceedings of the National Academy of Sciences, U.S.A., 100, 3479-3484.

Irwin, W., Davidson, R. J., Lowe, M. J., Mock, B. J., Sorenson, J. A., \& Turski, P. A. (1996). Human amygdala activation detected with echo-planar functional magnetic resonance imaging. NeuroReport, 7, 1765-1769.

Junghöfer, M., Bradley, M. M., Elbert, T. R., \& Lang, P. J. (2001). Fleeting images: A new look at early emotion discrimination. Psychophysiology, 38, 175-178.

Kim, H., Somerville, L. H., Johnstone, T., Alexander, A. L., \& Whalen, P. J. (2003). Inverse amygdala and medial prefrontal cortex responses to surprised faces. NeuroReport, 14, 2317-2322. 
Kim, H., Somerville, L. H., Johnstone, T., Polis, S., Alexander, A. L., Shin, L. M., et al. (2004). Contextual modulation of amygdala responsivity to surprised faces. Journal of Cognitive Neuroscience, 16, 1730-1745.

Lang, P. J., Bradley, M. M., \& Cuthbert, B. N. (1997). International Affective Picture System (IAPS): Technical manual and affective ratings. Gainsville, FL: The Center for Research in Psychophysiology, University of Florida.

MacKinnon, D. P., Lockwood, C. M., Hoffman, J. M., West, S. G., \& Sheets, V. (2002). A comparison of methods to test mediation and other intervening variable effects. Psychological Methods, 7, 83-104.

MacPherson, S. E., Phillips, L. H., \& Della, S. S. (2002). Age, executive function, and social decision making: A dorsolateral prefrontal theory of cognitive aging. Psychology and Aging, 17, 598-609.

Mather, M., Canli, T., English, T., Whitfield, S., Wais, P., Ochsner, K., et al. (2004). Amygdala responses to emotionally valenced stimuli in older and younger adults. Psychological Science, 15, 259-263.

Mathews, A., \& McLeod, C. (2005). Cognitive vulnerability to emotional disorders. Annual Review of Clinical Psychology, 1, 167-195.

Mathews, A., Yiend, J., \& Lawrence, A. D. (2004). Individual differences in the modulation of fear-related brain activation by attentional control. Journal of Cognitive Neuroscience, 16, 1683-1694.

Mayberg, H. S. (1997). Limbic-cortical dysregulation: A proposed model of depression. Journal of Neuropsychiatry and Clinical Neurosciences, 9, 471-481.

Milad, M. R., \& Quirk, G. J. (2002). Neurons in medial prefrontal cortex signal memory for fear extinction. Nature, 420, 70-74.

Miller, E. K., \& Cohen, J. D. (2001). An integrative theory of prefrontal cortex function. Annual Review of Neuroscience, 24, 167-202.

Mogg, K., \& Bradley, B. P. (1999). Some methodological issues in assessing attentional biases for threatening faces in anxiety: A replication study using a modified version of the probe detection task. Behaviour Research and Therapy, 37, 595-604.

Mogg, K., McNamara, J., Powys, M., Rawlinson, H., Seiffer, A., \& Bradley, B. P. (2000). Selective attention to threat: A test of two cognitive models of anxiety. Cognition and Emotion, 14, 375-399.

Öhman, A., Flykt, A., \& Esteves, F. (2001). Emotion drives attention: Detecting the snake in the grass. Journal of Experimental Psychology: General, 130, 466-478.

Phan, K. L., Taylor, S. F., Welsh, R. C., Ho, S. H., Britton, J. C., \& Liberzon, I. (2004). Neural correlates of individual ratings of emotional salience: A trial-related fMRI study. Neuroimage, 21, 768-780.

Phelps, E. A., Delgado, M. R., Nearing, K. I., \& LeDoux, J. E. (2004). Extinction learning in humans: Role of the amygdala and vmPFC. Neuron, 43, 897-905.

Phelps, E. A., O’Connor, K. J., Gatenby, J. C., Gore, J. C., Grillon, C., \& Davis, M. (2001). Activation of the left amygdala to a cognitive representation of fear. Nature Neuroscience, 4, 437-441.

Raz, N., Gunning, F. M., Head, D., Dupuis, J. H., McQuain, J., Briggs, S. D., et al. (1997). Selective aging of the human cerebral cortex observed in vivo: Differential vulnerability of the prefrontal gray matter. Cerebral Cortex, 7, 268-282.

Raz, N., Gunning-Dixon, F., Head, D., Rodrigue, K. M., Williamson, A., \& Acker, J. D. (2004). Aging, sexual dimorphism, and hemispheric asymmetry of the cerebral cortex: Replicability of regional differences in volume. Neurobiology of Aging, 25, 377-396.
Robinson, M. D., Storbeck, J., Meier, B. P., \& Kirkeby, B. S. (2004). Watch out! That could be dangerous: Valencearousal interactions in evaluative processing. Personality and Social Psychology Bulletin, 30, 1472-1484.

Robinson, M. D., Vargas, P. T., Tamir, M., \& Solberg, E. C. (2004). Using and being used by categories-The case of negative evaluations and daily well-being. Psychological Science, 15, 521-526.

Rolls, E. T. (1999). The brain and emotion. New York: Oxford University Press.

Ryff, C. D. (1989). Happiness is everything, or is it? Explorations on the meaning of psychological well-being. Journal of Personality and Social Psychology, 57, 1069-1081.

Ryff, C. D., Dienberg, L. G., Urry, H. L., Muller, D., Rosenkranz, M. A., Friedman, E. M., et al. (2006). Psychological well-being and ill-being: Do they have distinct or mirrored biological correlates? Psychotherapy and Psychosomatics, 75, 85-95.

Ryff, C. D., Singer, B. H., \& Love, G. D. (2004). Positive health: Connecting well-being with biology. Philosophical Transactions of the Royal Society of London, Series B, Biological Sciences, 359, 1383-1394.

Schaefer, S. M., Jackson, D. C., Davidson, R. J., Aguirre, G. K., Kimberg, D. Y., \& Thompson-Schill, S. L. (2002). Modulation of amygdalar activity by the conscious regulation of negative emotion. Journal of Cognitive Neuroscience, 14, 913-921.

Shackman, A. J., Maxwell, J. S., Springborn, K. D., Greischar, L. L., Pizzagalli, D. A., Coan, J. A., et al. (2005). Trait anxiety promotes threat-evoked negative affect through reduced ventromedial PFC inhibition. Poster presented at the 12th Annual Meeting of the Cognitive Neuroscience Society, New York, NY, USA.

Simpson, J. R. J., Drevets, W. C., Snyder, A. Z., Gusnard, D. A., \& Raichle, M. E. (2001). Emotion-induced changes in human medial prefrontal cortex: II. During anticipatory anxiety. Proceedings of the National Academy of Sciences, U.S.A., 98, 688-693.

Spielberger, C. D., Gorsuch, R. L., Lushene, R. E., Vagg, P. R., \& Jacobs, G. A. (1983). Manual for the state-trait anxiety inventory. Palo Alto, CA: Consulting Psychologists Press.

Taylor, S. E. (1991). Asymmetrical effects of positive and negative events: The mobilization-minimization hypothesis. Psychological Bulletin, 110, 67-85.

Tessitore, A., Hariri, A. R., Fera, F., Smith, W. G., Das, S., Weinberger, D. R., et al. (2005). Functional changes in the activity of brain regions underlying emotion processing in the elderly. Psychiatry Research: Neuroimaging, 139, 9-18.

Urry, H. L., van Reekum, C. M., Johnstone, T., Kalin, N. H., Thurow, M. E., Schaefer, H. S., et al. (2006). Amygdala and ventromedial prefrontal cortex are inversely coupled during regulation of negative affect and predict the diurnal pattern of cortisol secretion among older adults. Journal of Neuroscience, 26, 4415-4425.

Watson, D., \& Clark, L. A. (1991). Self-versus peer ratings of specific emotional traits: Evidence of convergent and discriminant validity. Journal of Personality and Social Psychology, 60, 927-940.

Whalen, P. J. (1998). Fear, vigilance, and ambiguity: Initial neuroimaging studies of the human amygdala. Current Directions in Psychological Science, 7, 177-187.

Williams, J. M., Mathews, A., \& MacLeod, C. (1996). The emotional Stroop task and psychopathology. Psychological Bulletin, 120, 3-24.

Williams, L. M., Phillips, M. L., Brammer, M. J., Skerrett, D., Lagopoulos, J., Rennie, C., et al. (2001). Arousal dissociates amygdala and hippocampal fear responses: Evidence from simultaneous fMRI and skin conductance recording. Neuroimage, 14, 1070-1079. 